\title{
Qualitative behavior of a rational difference equation $\gamma_{n+1}=\frac{y_{n}+y_{n-1}}{p+y_{y} h-1}$
}

Xiao Qian ${ }^{*}$ and Shi Qi-hong

* Correspondence: xiaoxiao_xq168@163.com Department of Basic Courses, Hebei Finance University, Baoding 071000, China

\section{Abstract}

This article is concerned with the following rational difference equation $y_{n+1}=\left(y_{n}+\right.$ $\left.y_{n-1}\right) /\left(p+y_{n} y_{n-1}\right)$ with the initial conditions; $y_{-1}, y_{0}$ are arbitrary positive real numbers, and $p$ is positive constant. Locally asymptotical stability and global attractivity of the equilibrium point of the equation are investigated, and non-negative solution with prime period two cannot be found. Moreover, simulation is shown to support the results.

Keywords: Global stability attractivity, solution with prime period two, numerical simulation

\section{Introduction}

Difference equations are applied in the field of biology, engineer, physics, and so on [1]. The study of properties of rational difference equations has been an area of intense interest in the recent years [6,7]. There has been a lot of work deal with the qualitative behavior of rational difference equation. For example, Çinar [2] has got the solutions of the following difference equation:

$$
x_{n+1}=\frac{a x_{n-1}}{1+b x_{n} x_{n-1}}
$$

Karatas et al. [3] gave that the solution of the difference equation:

$$
x_{n+1}=\frac{x_{n-5}}{1+x_{n-2} x_{n-5}} \text {. }
$$

In this article, we consider the qualitative behavior of rational difference equation:

$$
y_{n+1}=\frac{y_{n}+y_{n-1}}{p+y_{n} y_{n-1}}, \quad n=0,1, \ldots,
$$

with initial conditions $y_{-1}, y_{0} \in(0,+\infty), p \in R^{+}$.

\section{Preliminaries and notation}

Let us introduce some basic definitions and some theorems that we need in what follows.

Lemma 1. Let $I$ be some interval of real numbers and

$$
f: I^{2} \rightarrow I
$$

(C) 2011 Qian and Qi-hong; licensee Springer. This is an Open Access article distributed under the terms of the Creative Commons Attribution License (http://creativecommons.org/licenses/by/2.0), which permits unrestricted use, distribution, and reproduction in any medium, provided the original work is properly cited. 
be a continuously differentiable function. Then, for every set of initial conditions, $x_{-k}$, $x_{-k+1}, \ldots, x_{0} \in I$ the difference equation

$$
x_{n+1}=f\left(x_{n}, x_{n-1}\right), \quad n=0,1, \ldots
$$

has a unique solution $\left\{x_{n}\right\}_{n=-k}^{\infty}$.

Definition 1 (Equilibrium point). A point $\bar{x} \in I$ is called an equilibrium point of Equation 2, if

$$
\bar{x}=f(\bar{x}, \bar{x})
$$

Definition 2 (Stability). (1) The equilibrium point $\bar{x}$ of Equation 2 is locally stable if for every $\varepsilon>0$, there exists $\delta>0$, such that for any initial data $x_{-k}, x_{-k+1}, \ldots, x_{0} \in I$, with

$$
\left|x_{-k}-\bar{x}\right|+\left|x_{-k+1}-\bar{x}\right|+\cdots+\left|x_{0}-\bar{x}\right|<\delta,
$$

we have $\left|x_{n}-\bar{x}\right|<\varepsilon$, for all $n \geq-k$.

(2) The equilibrium point $\bar{x}$ of Equation 2 is locally asymptotically stable if $\bar{x}$ is locally stable solution of Equation 2, and there exists $\gamma>0$, such that for all $x_{-k}, x_{-k+1}$, ..., $x_{0} \in I$, with

$$
\left|x_{-k}-\bar{x}\right|+\left|x_{-k+1}-\bar{x}\right|+\cdots+\left|x_{0}-\bar{x}\right|<\gamma,
$$

we have

$$
\lim _{n \rightarrow \infty} x_{n}=\bar{x}
$$

(3) The equilibrium point $\bar{x}$ of Equation 2 is a global attractor if for all $x_{-k}, x_{-k+1}, \ldots$, $x_{0} \in I$, we have $\lim _{n \rightarrow \infty} x_{n}=\bar{x}$.

(4) The equilibrium point $\bar{x}$ of Equation 2 is globally asymptotically stable if $\bar{x}$ is locally stable and $\bar{x}$ is also a global attractor of Equation 2 .

(5) The equilibrium point $\bar{x}$ of Equation 2 is unstable if $\bar{x}$ is not locally stable.

Definition 3 The linearized equation of (2) about the equilibrium $\bar{x}$ is the linear difference equation:

$$
y_{n+1}=\sum_{i=0}^{k} \frac{\partial f(\bar{x}, \bar{x}, \ldots, \bar{x})}{\partial x_{n-i}} y_{n-i}
$$

Lemma 2 [4]. Assume that $p_{1}, p_{2} \in R$ and $k \in\{1,2, \ldots\}$, then

$$
\left|p_{1}\right|+\left|p_{2}\right|<1,
$$

is a sufficient condition for the asymptotic stability of the difference equation

$$
x_{n+1}-p_{1} x_{n}-p_{2} x_{n-1}=0, \quad n=0,1, \ldots
$$

Moreover, suppose $p_{2}>0$, then, $\left|p_{1}\right|+\left|p_{2}\right|<1$ is also a necessary condition for the asymptotic stability of Equation 4 .

Lemma 3 [5]. Let $g:[p, q]^{2} \rightarrow[p, q]$ be a continuous function, where $p$ and $q$ are real numbers with $p<q$ and consider the following equation:

$$
x_{n+1}=g\left(x_{n}, x_{n-1}\right), \quad n=0,1, \ldots
$$

Suppose that $g$ satisfies the following conditions: 
(1) $g(x, y)$ is non-decreasing in $x \in[p, q]$ for each fixed $y \in[p, q]$, and $g(x, y)$ is nonincreasing in $y \in[p, q]$ for each fixed $x \in[p, q]$.

(2) If $(m, M)$ is a solution of system

$M=g(M, m)$ and $m=g(m, M)$,

then $M=m$.

Then, there exists exactly one equilibrium $\bar{x}$ of Equation 5 , and every solution of Equation 5 converges to $\bar{x}$.

\section{The main results and their proofs}

In this section, we investigate the local stability character of the equilibrium point of Equation 1. Equation 1 has an equilibrium point

$$
\bar{x}=\left\{\begin{array}{l}
0, \quad p \geq 2 \\
0, \sqrt{2-p} \quad p<2
\end{array} .\right.
$$

Let $f:(0, \infty)^{2} \rightarrow(0, \infty)$ be a function defined by

$$
f(u, v)=\frac{u+v}{p+u v}
$$

Therefore, it follows that

$$
f_{u}(u, v)=\frac{p-v^{2}}{(p+u v)^{2}}, \quad f_{v}(u, v)=\frac{p-u^{2}}{(p+u v)^{2}} .
$$

Theorem 1. (1) Assume that $p>2$, then the equilibrium point $\bar{x}=0$ of Equation 1 is locally asymptotically stable.

(2) Assume that $0<p<2$, then the equilibrium point $\bar{x}=\sqrt{2-p}$ of Equation 1 is locally asymptotically stable, the equilibrium point $\bar{x}=0$ is unstable.

Proof. (1) when $\bar{x}=0$,

$$
f_{u}(\bar{x}, \bar{x})=\frac{1}{p}, \quad f_{v}(\bar{x}, \bar{x})=\frac{1}{p} .
$$

The linearized equation of (1) about $\bar{x}=0$ is

$$
y_{n+1}-\frac{1}{p} y_{n}-\frac{1}{p} y_{n-1}=0
$$

It follows by Lemma 2, Equation 7 is asymptotically stable, if $p>2$.

(2) when $\bar{x}=\sqrt{2-p}$,

$$
f_{u}(\bar{x}, \bar{x})=\frac{p-1}{2}, \quad f_{v}(\bar{x}, \bar{x})=\frac{p-1}{2} .
$$

The linearized equation of (1) about $\bar{x}=\sqrt{2-p}$ is

$$
y_{n+1}-\frac{p-1}{2} y_{n}-\frac{p-1}{2} y_{n-1}=0 .
$$

It follows by Lemma 2, Equation 8 is asymptotically stable, if

$$
\left|\frac{p-1}{2}\right|+\left|\frac{p-1}{2}\right|<1,
$$


Therefore,

$$
0<p<2 .
$$

Equilibrium point $\bar{x}=0$ is unstable, it follows from Lemma 2. This completes the proof.

Theorem 2. Assume that $v_{0}^{2}<p<u_{0}^{2}$, the equilibrium point $\bar{x}=0$ and $\bar{x}=\sqrt{2-p}$ of Equation 1 is a global attractor.

Proof. Let $p, q$ be real numbers and assume that $g:[p, q]^{2} \rightarrow[p, q]$ be a function defined by $g(u, v)=\frac{u+v}{p+u v}$, then we can easily see that the function $g(u, v)$ increasing in $u$ and decreasing in $v$.

Suppose that $(m, M)$ is a solution of system

$M=g(M, m)$ and $m=g(m, M)$.

Then, from Equation 1

$$
M=\frac{M+m}{p+M m}, \quad m=\frac{M+m}{p+M m} .
$$

Therefore,

$$
\begin{aligned}
& p M+M^{2} m=M+m, \\
& p m+M m^{2}=M+m .
\end{aligned}
$$

Subtracting Equation 10 from Equation 9 gives

$$
(p+M m)(M-m)=0 .
$$

Since $p+M m \neq 0$, it follows that

$$
M=m \text {. }
$$

Lemma 3 suggests that $\bar{x}$ is a global attractor of Equation 1 and then, the proof is completed.

Theorem 3. (1) has no non-negative solution with prime period two for all $p \in R^{+}$.

Proof. Assume for the sake of contradiction that there exist distinctive non-negative real numbers $\phi$ and $\psi$, such that

$$
\ldots, \varphi, \psi, \varphi, \psi, \ldots
$$

is a prime period-two solution of (1).

$\phi$ and $\psi$ satisfy the system

$$
\begin{aligned}
& \varphi(p+\varphi \psi)=\varphi+\psi, \\
& \psi(p+\varphi \psi)=\psi+\varphi,
\end{aligned}
$$

Subtracting Equation 11 from Equation 12 gives

$$
(\varphi-\psi)(p+\varphi \psi)=0
$$

so $\phi=\psi$, which contradicts the hypothesis $\phi \neq \psi$. The proof is complete. 


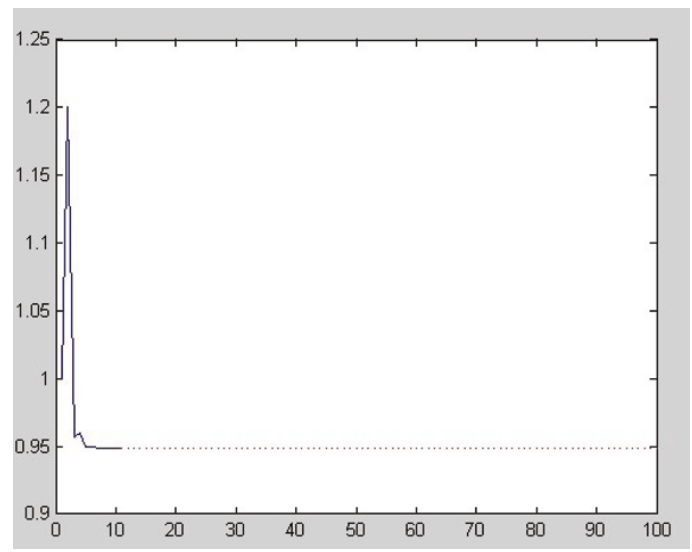

Figure 1 Plot of $x(n+1)=(x(n)+x(n-1)) /\left(1.1+x(n)^{*} x(n-1)\right)$. This figure shows the solution of $y_{n+1}=\frac{y_{n}+y_{n-1}}{1.1+y_{n} y_{n-1}}$, where $x_{0}=1, x_{1}=1.2$

\section{Numerical simulation}

In this section, we give some numerical simulations to support our theoretical analysis. For example, we consider the equation:

$$
\begin{gathered}
y_{n+1}=\frac{y_{n}+y_{n-1}}{1.1+y_{n} y_{n-1}} \\
y_{n+1}=\frac{y_{n}+y_{n-1}}{1.5+y_{n} y_{n-1}} \\
y_{n+1}=\frac{y_{n}+y_{n-1}}{5+y_{n} y_{n-1}}
\end{gathered}
$$

We can present the numerical solutions of Equations 13-15 which are shown, respectively in Figures 1, 2 and 3. Figure 1 shows the equilibrium point $\bar{x}=\sqrt{2-1.1}$ of Equation 13 is locally asymptotically stable with initial data $x_{0}=1, x_{1}=1.2$. Figure 2 shows the equilibrium point $\bar{x}=\sqrt{2-1.5}$ of Equation 14 is locally asymptotically

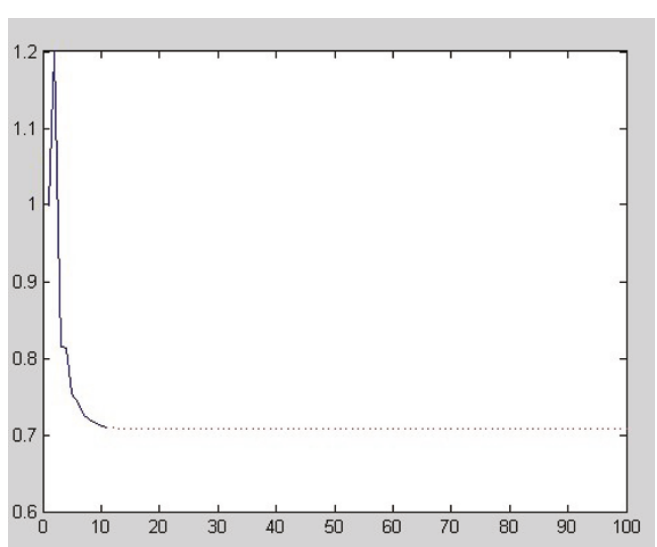

Figure 2 Plot of $x(n+1)=(x(n)+x(n-1)) /(1.5+x(n) * x(n-1))$. This figure shows the solution of $y_{n+1}=\frac{y_{n}+y_{n-1}}{1.5+y_{n} y_{n-1}}$, where $x_{0}=1, x_{1}=1.2$ 


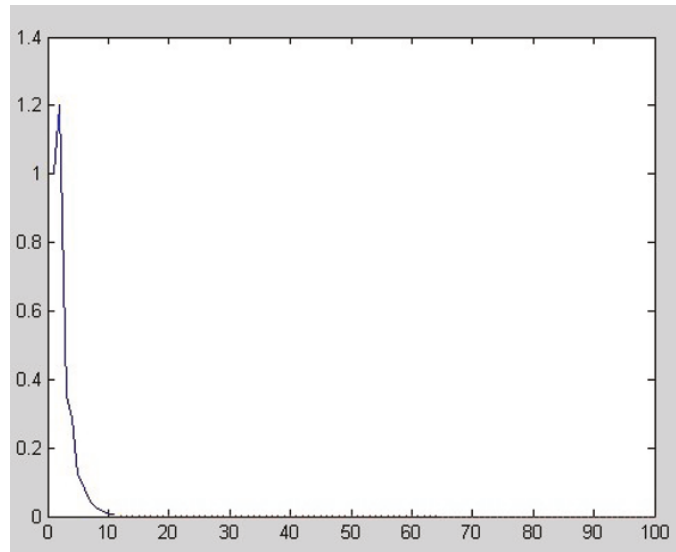

Figure 3 Plot of Plot of $x(n+1)=(x(n)+x(n-1)) /\left(5+x(n)^{*} x(n-1)\right)$. This figure shows the solution of $y_{n+1}=\frac{y_{n}+y_{n-1}}{5+y_{n} y_{n-1}}$, where $x_{0}=1, x_{1}=1.2$

stable with initial data $x_{0}=1, x_{1}=1.2$. Figure 3 shows the equilibrium point $\bar{x}=0$ of Equation 15 is locally asymptotically stable with initial data $x_{0}=1, x_{1}=1.2$.

\section{Authors' contributions}

Xiao Qian carried out the theoretical proof and drafted the manuscript. Shi Qi-hong participated in the design and coordination. All authors read and approved the final manuscript.

\section{Competing interests}

The authors declare that they have no competing interests.

Received: 10 February 2011 Accepted: 3 June 2011 Published: 3 June 2011

\section{References}

1. Berezansky L, Braverman E, Liz E: Sufficient conditions for the global stability of nonautonomous higher order difference equations. J Diff Equ Appl 2005, 11(9):785-798.

2. Çinar C: On the positive solutions of the difference equation $x_{n+1}=a x_{n-1} / 1+b x_{n} x_{n-1}$. Appl Math Comput 2004, 158(3):809-812.

3. Karatas R, Cinar C, Simsek D: On positive solutions of the difference equation $x_{n+1}=x_{n-5} / 1+x_{n-2} x_{n-5}$. Int J Contemp Math Sci 2006, 1(10):495-500

4. Li W-T, Sun H-R: Global attractivity in a rational recursive sequence. Dyn Syst Appl 2002, 3(11):339-345.

5. Kulenovic MRS, Ladas G: Dynamics of Second Order Rational Difference Equations with Open Problems and Conjectures. Chapman \& Hall/CRC Press; 2001

6. Elabbasy EM, El-Metwally $H$, Elsayed EM: On the difference equation $x_{n+1}=a x_{n}-b x_{n} /\left(c x_{n}-d x_{n-1}\right)$. Adv Diff Equ 2006, $1-10$.

7. Memarbashi R: Sufficient conditions for the exponential stability of nonautonomous difference equations. App/ Math Lett 2008, 3(21):232-235.

doi:10.1186/1687-1847-2011-6

Cite this article as: Qian and Qi-hong: Qualitative behavior of a rational difference equation . Advances in Difference Equations 2011 2011:6. 